\title{
Scattering Correction Based on Regularization De-Convolution for Cone-Beam CT
}

\author{
Rui-Ju Yan and Shi-Peng Xie ${ }^{+}$ \\ College of Telecommunications and Information Engineering, Nanjing University of Posts and \\ Telecommunications, Nanjing, Jiangsu 210003, China
}

\begin{abstract}
In Cone-Beam CT (CBCT) imaging systems, the scattering phenomenon has a significant impact on the reconstructed image and is a long-lasting research topic on CBCT. In this paper, we propose a simple, novel and fast approach for mitigating scatter artifacts and increasing the image contrast in CBCT, belonging to the category of convolution-based method in which the projected data is de-convolved with a convolution kernel. A key step in this method is how to determine the convolution kernel. Compared with existing methods, the estimation of convolution kernel is based on bi-11-12-norm regularization imposed on both the intermediate the known scatter contaminated projection images $g$ and the convolution kernel. Our approach can reduce the scatter artifacts from 12.930 to 2.133 .
\end{abstract}

Keywords: scatter correction, cone-beam CT (CBCT), convolution kernel, regularization.

\section{Introduction}

Computerized Tomography (CT) is a technique for imaging the cross sections of an object using a series of x-ray measurements taken from different angles around the object. An x-ray system with a large-area detector, which is commonly used for cone-beam computed tomography (CBCT), is more susceptible to cupping artifacts generated by scatter and beam hardening. The presence of scatter in the projection images leads to reduced low-contrast sensitivity, artifacts such as dark bands behind dense objects, and slowly varying CT number nonlinearities known as cupping artifacts in the reconstructed 3D images. Beam hardening is also known to lead to image cupping artifacts. X-ray scatter may lead to additional artifacts, which is one of the most challenging problems in CBCT. In the past 20 years, most correction methods that have been developed to reduce the artifacts focus on reducing the scattered photons on the projective image. Various correction methods using software-based [1-3], hardware-based [4-6], or combined hybrid approaches [7-12] have been proposed in the literature. The effect of software-based convolution and de-convolution correction methods depends on whether the chosen conditions which the point spread function and the actual experiment conditions of X-rays in the actual experiment are the same. When the experimental conditions change, we can not guarantee the suppress effect of scatting, therefore the limitation of application is relatively large.

\section{Method}

The scatter correction algorithm with the convolution is divided into the following steps:

\footnotetext{
${ }^{+}$Corresponding author. Tel.: +18252065157.

E-mail address: xie@njupt.edu.cn.
} 


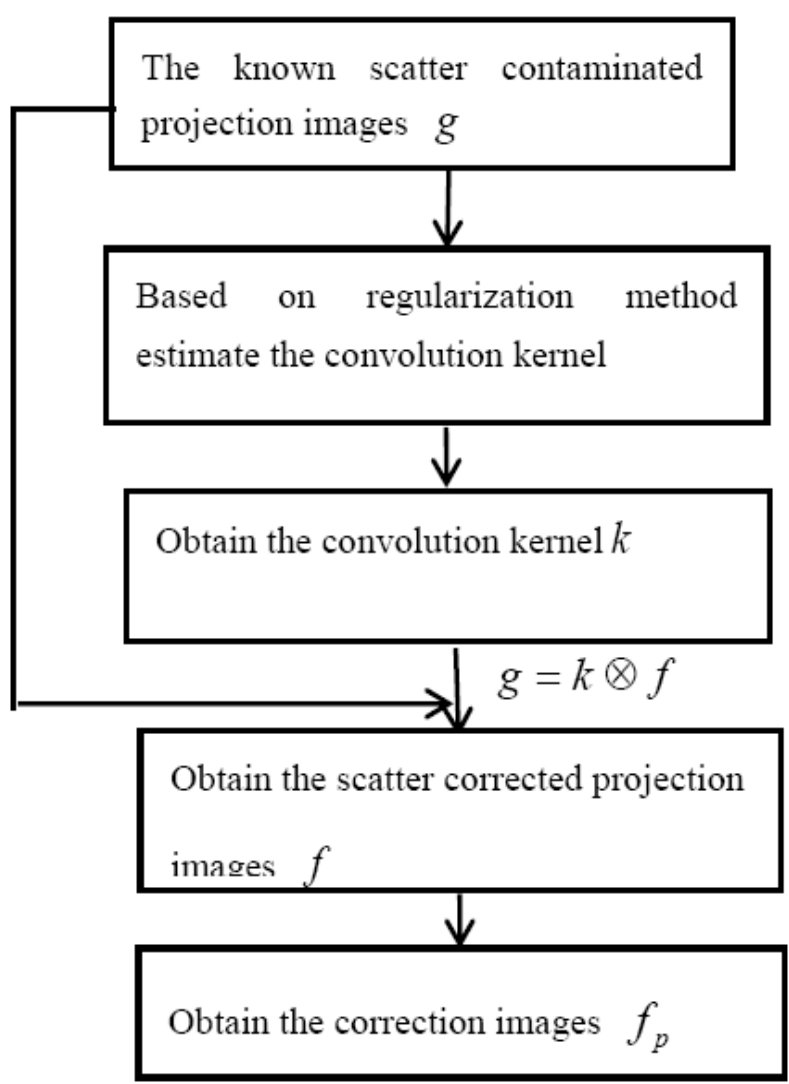

Fig. 1: The process of the proposed cupping artifact correction method using de-convolution method.

\section{Result}

The phantom is the projected data which is de-convolved with a convolution kernel. Fig. 2 and Fig. 4 shows the reconstructed images.

The 1D horizontal profile with and without scatter signals can be seen in Fig. 3and Fig. 5.

For a quantitative analysis of the reconstructed image, we measured the image contrast and the magnitude of cupping in Fig. 2 (a) and Fig. 4(a)(c). Our approach can increase the image CNR from 5.61 to 7.35 and reduce the artifact from $12.930 \%$ to $2.133 \%$. These artifacts are significantly suppressed when the proposed method is used with convolution

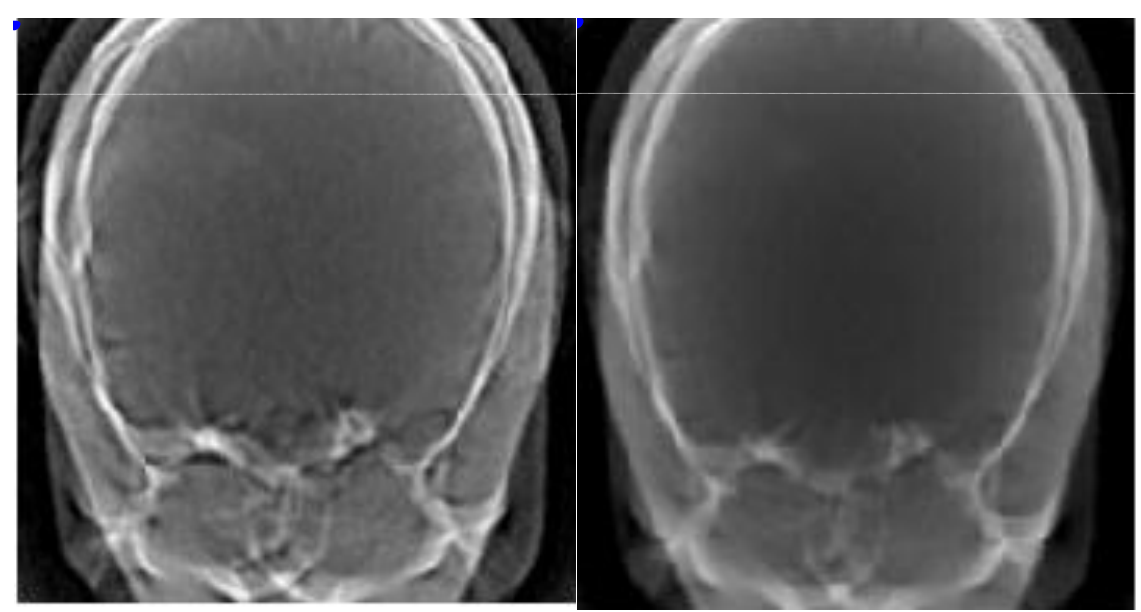

(a)

(b)

Fig. 2: Image reconstructions of the phantom. (a) CBCT without scatter correction; (b) scatter correction using the de-convolution method. 


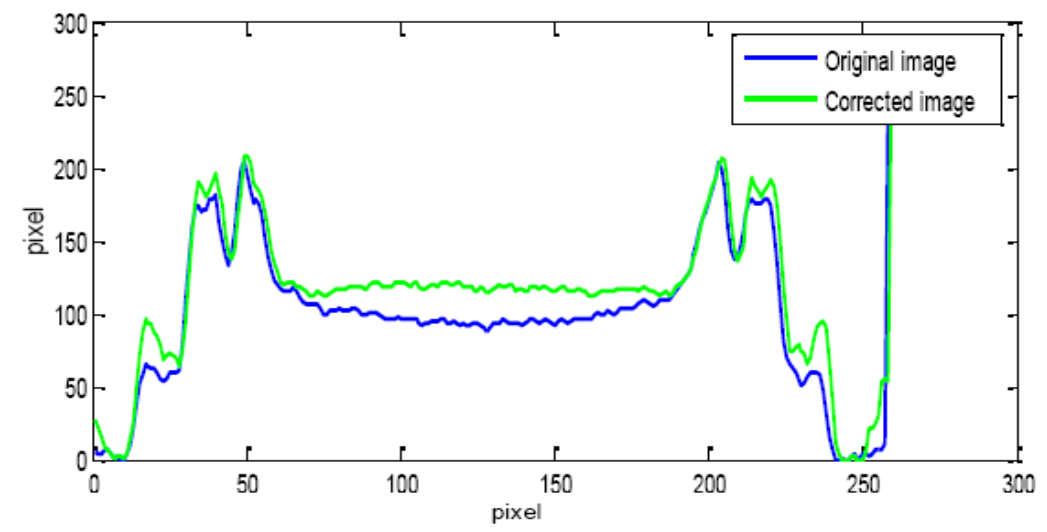

Fig. 3: The 1D horizontal profile of the measured and estimated scatter signals on the phantom: The column is the profiles of the projection image which is at row 26 in Fig. 2.

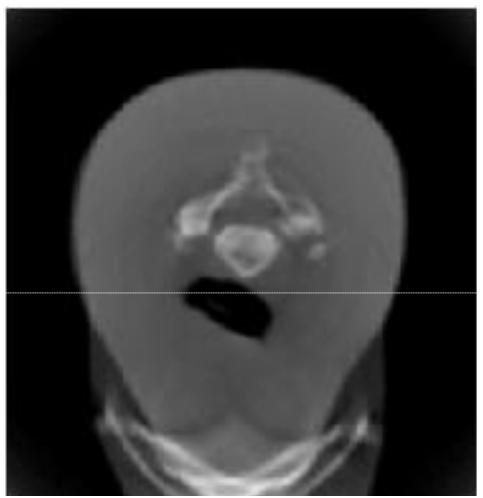

(a)

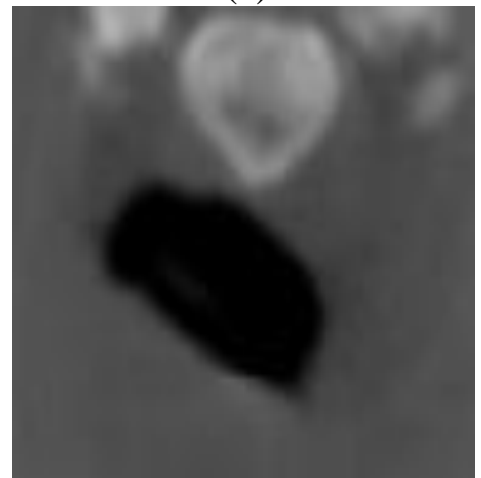

(c)

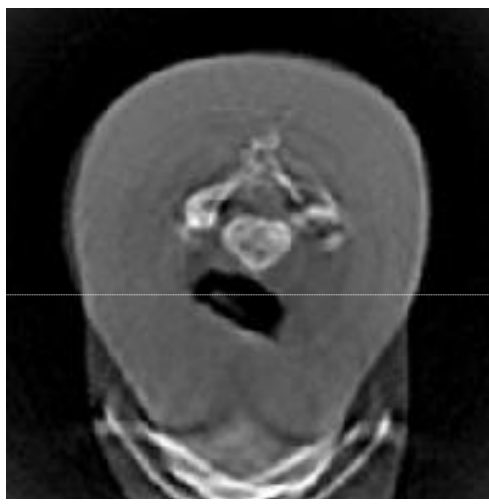

(b)

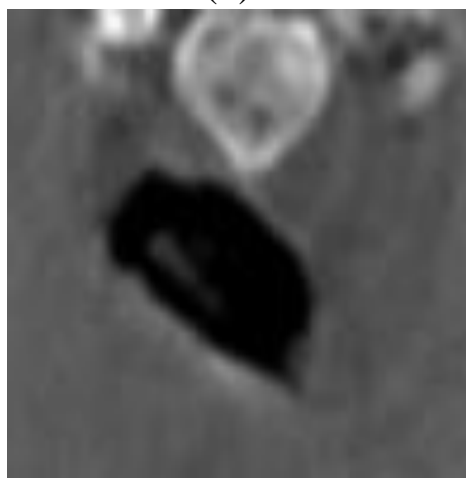

(d)

Fig. 4: Image reconstructions of the phantom. (a)(b) CBCT without scatter correction; (b) (d) Scatter correction using the de-convolution method.

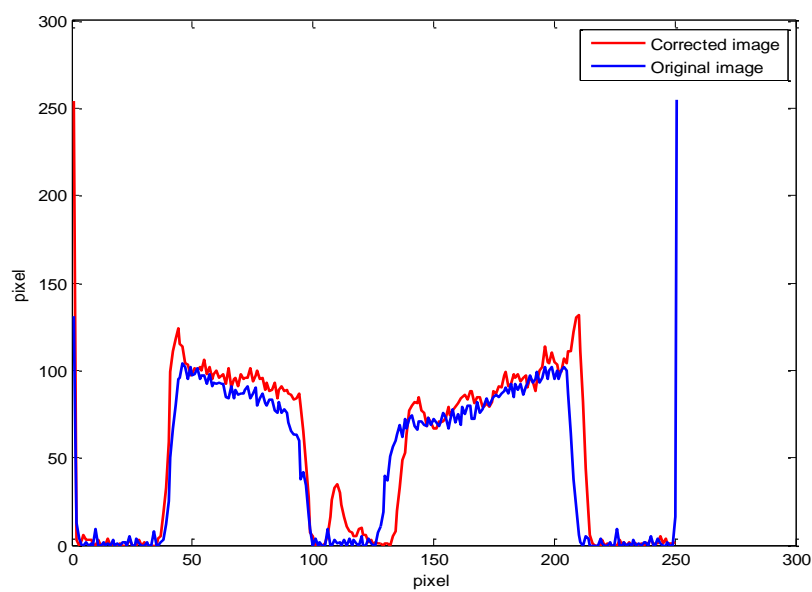

Fig. 5: The 1D horizontal profile of the measured and estimated scatter signals on the phantom: The column is the profiles of the projection image which is at row 127 in Fig. 4. 


\section{Acknowledgments}

This work was supported by the National Natural Science Foundation of China (Grant NO. 11547155), National Natural Science Foundation of Jiangsu Province (Grant NO. BK20130883) and the NUPTSF (Grant No. NY213011 and NO. 214026).

\section{References}

[1] Seibert J.A; An analytical model of the scattered radiation distribution in diagnostic radiology, Med. Phys. 15(5):721-725 (1988).

[2] Floyd, C E, et al. "Scatter compensation for digital chest radiography using maximum likelihood expectation maximization.." Investigative Radiology 28(5):427-433(1993)

[3] Honda M, Kikuchi K, Komatsu K. Method for estimating the intensity of scattered radiation using a scatter generation model.[J]. Medical Physics. 18(2), 219 - 226 (1991)

[4] J. S. Maltz et al., "Focused beam-stop array for the measurement of scatter in megavoltage portal and cone beam CT imaging,” Med. Phys. 35, 2452 - 2462, (2008).

[5] L. Ouyang et al., "Optimization of a moving blocker system for cone beam computed tomography scatter correction,” Med. Phys. 41(6), 125(2014).

[6] L. Zhu, N. R. Bennett, and R. Fahrig, "Scatter correction method for X-ray CT using primary modulation: Theory and preliminary results,” IEEE Trans. Med. Imaging 25(12), 1573 - 1587 (2006).

[7] Zhu L, Bennett N R, Fahrig R. Scatter correction method for X-ray CT using primary modulation: theory and preliminary results.[J]. IEEE Transactions on Medical Imaging, 25(12), 1573-1587 (2006).

[8] Zhu L, Wang J, Xing L. Noise suppression in scatter correction for cone-beam CT.[J]. Medical Physics, 36(3),741-752 (2009).

[9] Shi-Peng X,Li-Min.Scatter correction for cone beam CT using self-adaptive scatter kernel superposition[J]. Chinese physics C,2012,36(6):566-572.MLA

[10] Li Q, Yan B, Li L, et al. Scatter correction algorithm for cone-beam computed tomography based on convolution and total variation model[J]. High Power Laser \& Particle Beams, 24(9):2235-2238(2012).

[11] Xie S P,Luo L M.Scatter correction for cone beam CT using attenuation baffle[J].Dianzi Xuebao(Acta Electronica Sinica),2011,39(7):1708-1711(2011).

[12] Shipeng Xie,Chunming Li,Haibo Li,Qi Ge,"A level set method for cupping artifact correction in cone-beam CT,"Medical Physics,2015,42(8):4888-4895. 